\title{
Steen Hyldgaard Christensen, Martin Meganck et Bernard Delahousse (dir.), Philosophy in Engineering
}

Aarhus, Danemark : Academica, 2007

\section{Daniel Atlan}

\section{OpenEdition}

\section{Journals}

Édition électronique

URL : http://journals.openedition.org/asp/310

DOI : $10.4000 /$ asp.310

ISBN : 978-2-8218-0404-3

ISSN : 2108-6354

\section{Éditeur}

Groupe d'étude et de recherche en anglais de spécialité

\section{Édition imprimée}

Date de publication : 1 décembre 2007

Pagination : 195-196

ISSN : 1246-8185

\section{Référence électronique}

Daniel Atlan, « Steen Hyldgaard Christensen, Martin Meganck et Bernard Delahousse (dir.), Philosophy in Engineering », ASp [En ligne], 51-52 | 2007, mis en ligne le 31 décembre 2009, consulté le 22 mars 2021. URL : http://journals.openedition.org/asp/310 ; DOI : https://doi.org/10.4000/asp.310

Ce document a été généré automatiquement le 22 mars 2021.

Tous droits réservés 


\section{Steen Hyldgaard Christensen,} Martin Meganck et Bernard Delahousse (dir.), Philosophy in Engineering

Aarhus, Danemark : Academica, 2007

Daniel Atlan

\section{RÉFÉRENCE}

Christensen, Steen Hyldgaard, Martin Meganck et Bernard Delahousse (dir.). 2007.

Philosophy in Engineering. Aarhus, Danemark : Academica, 430 p. 
1 Socrate et Erasme seraient fiers de cet ouvrage dont le financement a été assuré par les fonds européens éponymes. Vingtquatre enseignants et chercheurs de quinze pays membres de l'Union ont contribué à la rédaction et à la mise en forme de ce livre qui se veut un support à l'enseignement de la philosophie de l'ingénierie dans un premier cycle universitaire. Mais que le lecteur potentiel ne soit pas détourné de sa lecture s'il a d'autres intérêts que ce seul enseignement. Cet ouvrage est d'une grande richesse et ouvre de nombreuses pistes de réflexion (et d'action), tant pour l'enseignement que pour la pratique de l'ingénieur, mais aussi pour la compréhension du monde contemporain.

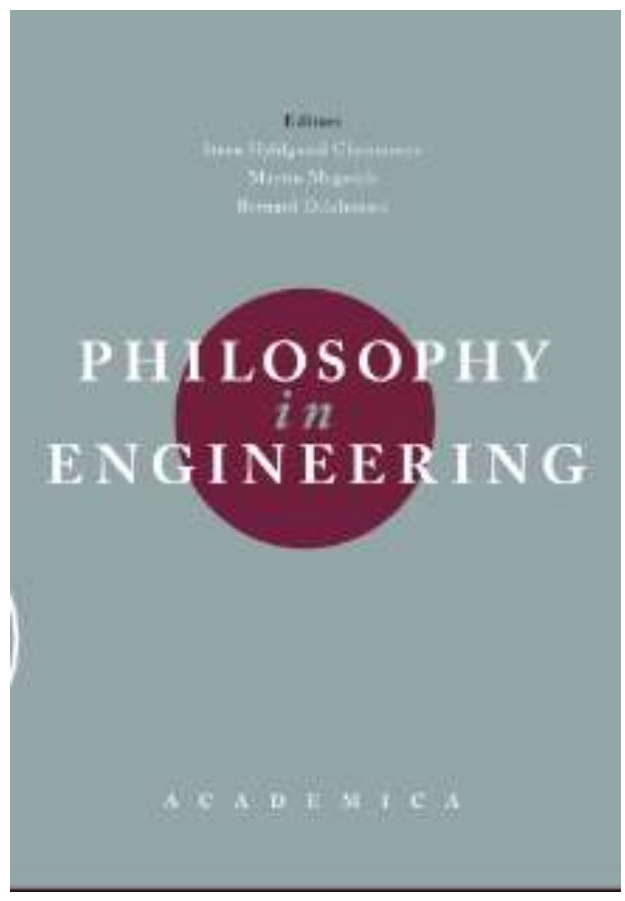

2 Philosophy in Engineering est organisé d'une manière très didactique: chacune des quatre grandes parties est précédée d'une courte introduction qui en présente le thème et les enjeux: "Formation des ingénieurs dans l'histoire [depuis la Renaissance], Philosophie et épistémologie de la pratique et de la science de l'ingénieur, Éthique et ingénierie, Rôles et statut social de l'ingénieur dans la société de la connaissance ».

3 Les vingt chapitres peuvent se lire indépendamment les uns des autres. Néanmoins, le travail éditorial assurant l'homogénéité de l'ensemble est remarquable. Il n'y a quasiment pas de redites et, de plus, le lecteur découvre des renvois et des références entre les chapitres qui tissent des liens tout au long du livre et en assurent l'unité intellectuelle.

Dans chaque grande partie, le lecteur trouvera plusieurs exemples concrets, convenablement étayés par des documents, et donc susceptibles de constituer autant d'études de cas pour l'enseignant. Ces exemples éclairent le débat et mettent en perspective les questions clés.

Le livre se termine par un glossaire, où les termes sont définis de façon très claire, et par un index dont on peut toutefois regretter le caractère quelque peu incomplet, surtout pour ce qui est des noms propres.

Un des concepts clés qui structurent cet ouvrage est celui de «Bildung ${ }^{1}$ ", terme allemand signifiant éducation et formation, défini dans le glossaire comme «le processus et le produit de la formation d'une personne, d'un groupe ou d'une société dans le domaine des idées, des normes et des comportements ». Par un effet de miroir, Philosophy in Engineering est un outil pédagogique qui, à mon sens, peut contribuer de façon décisive à la formation des ingénieurs d'aujourd'hui. Ce type d'enseignement est peu répandu en France, que ce soit dans les premiers cycles techniques, dans les universités ou dans les Grandes Écoles. Les enseignants intéressés trouveront donc dans ce livre matière à bâtir une intervention pertinente. 
7 Quel est le public visé par cet ouvrage ? En quoi peut-il être utile à des enseignants de langue dans des universités en 2007 ? Cette dernière question reçoit plusieurs réponses au fil du livre.

8 Anders Buch, au chapitre 8, présente, en moins de vingt pages, un aperçu des théories de la connaissance et de l'apprentissage dans le domaine de l'ingénierie. L'enseignant de langue (ou de toute autre discipline) retrouvera là des thèmes bien connus mais dont la mise en œuvre dans un champ autre que le sien propre aide à la compréhension et à l'étude critique.

9 Dans le dernier chapitre, I. Turek et J. Miština convoquent les styles d'apprentissage dans leur réflexion sur les impacts de la mondialisation sur l'enseignement de l'ingénierie. Dans toute cette dernière partie de l'ouvrage, l'enseignant de langue trouvera des raisons de réfléchir à son rôle et à sa pratique dans la formation de techniciens et d'ingénieurs.

Ingénieurs et enseignants de langues se rejoignent ici dans les difficultés qu'ils rencontrent pour communiquer aux non spécialistes de leurs disciplines les enjeux liés à leur fonction, leurs attentes et leurs besoins. En ce sens, l'ouvrage établit un pont entre les deux communautés.

11 Enfin la deuxième partie, dans son ensemble, "Philosophie et épistémologie de la pratique et de la science de l'ingénieur", est centrée sur le statut spécifique de l'ingénierie par rapport aux sciences dures». Les didacticiens des disciplines y trouveront aussi matière à réflexion sur leur propre situation et leur propre pratique au regard de la discipline dont ils ou elles dépendent. S. H. Christensen et E. Kjølhede éclairent de façon convaincante la différence entre science et ingénierie. D'après eux, la conception (design) est l'activité centrale qui identifie l'ingénieur et qui le distingue du scientifique.

12 Sur ces questions, il est regrettable que les auteurs et les éditeurs n'aient pas fait appel aux apports de l'école française d'ethnométhodologie (B. Latour et M. Callon en particulier), ce qui aurait élargi la palette des références et contribué à la qualité des argumentaires.

13 Pour résumer, malgré cette réserve, l'ouvrage, d'un accès aisé grâce à sa conception, mérite largement d'être lu pour la pertinence des sujets qu'il aborde.

\section{NOTES}

1. Le titre anglais de la première section de l'ouvrage est « Occupational Bildung in Engineering: some Historical Examplars ». 


\section{AUTEURS}

DANIEL ATLAN

ArcelorMittal 